\title{
Efectividad de inducción de tolerancia inmune en niños con hemofilia A y aloanticuerpos neutralizantes
}

\author{
Immunotolerance induction effectivity in hemophilia A children and neutralizing \\ alloantibodies
}

\author{
Verónica Soto A. , Daniela Cortez S. ${ }^{a}$, Macarena González S. ${ }^{b}$ \\ apediatra Hematólogo. Hospital de Niños Roberto del Río. Santiago, Chile \\ BInterna de Medicina. Facultad de Medicina, Universidad de Chile. Santiago, Chile
}

Recibido: 9 de agosto de 2019; Aceptado: 25 de noviembre de 2019

¿Qué se sabe del tema que trata este estudio?

El conocimiento sobre la hemofilia, su tratamiento y sus complicaciones no es amplio dentro del mundo médico, dado que es una patología muy poco frecuente. La aparición de inhibidores es la complicación actual más severa y nuestro estudio relata una experiencia local y exitosa en su manejo, con el fin de despertar interés en ella.
¿Qué aporta este estudio a lo ya conocido?

El estudio publicado muestra que un complejo tratamiento, como es la Inducción de Tolerancia Inmune en pacientes con hemofilia A e inhibidores, es posible en nuestro país actualmente y con resultados comparables a los centros con los más altos estándares de atención de esta enfermedad en el mundo.

\section{Resumen}

El desarrollo de aloanticuerpos neutralizantes anti-factor VIII en hemofilia A es la complicación más seria relacionada al tratamiento. La inducción de tolerancia inmune (ITI) o inmunotolerancia es el único tratamiento que erradica inhibidores, permitiendo utilizar nuevamente factor VIII para el tratamiento o profilaxis de eventos hemorrágicos. Objetivo: reportar la experiencia en niños sometidos a inmunotolerancia en la red pública del país. Pacientes y Método: Análisis retrospectivo y descriptivo de 13 niños con Hemofilia A severa e inhibidores persistentes de alto título, que recibieron ITI y seguimiento completo. Se utilizó concentrado de FVIII plasmático en dosis de 70-180 UI/Kg/diarias, definiendo éxito como la negativización del inhibidor y recuperación de la vida media del FVIII. Resultados expresados en media (rango). Resultados: En 13 pacientes se identificó el inhibidor, a una edad de 17,6 meses (2-48), tras 35,2 días (9-112) de exposición a FVIII. Once pacientes $(84,6 \%)$ recuperaron la vida media del FVIII, tras 49,6 meses (26-70) de tratamiento. En los pacientes que respondieron, el título del inhibidor se negativizó en 7,3 meses (1-20). Conclusiones: En niños con hemofilia A e inhibidores persistentes de alto título, la ITI tiene un elevado éxito. Dado que el tiempo de respuesta es variable, la inmunotolerancia debe ser personalizada.
Palabras clave: Hemofilia A; factor VIII; aloanticuerpos neutralizantes; Inducción de inmunotolerancia 


\begin{abstract}
The development of anti-factor VIII neutralizing antibodies in hemophilia A is the most severe complication related to treatment. Immune tolerance induction (ITI) is the only known treatment for eradicating inhibitors. A successful ITI allows using factor VIII (FVIII) again for the treatment or prophylaxis of hemorrhagic events. Objective: To report the experience of pediatric patients who underwent ITI in the country's public health care network. Patients and Method: Retrospective and descriptive analysis of 13 pediatric patients with severe Hemophilia A and high-titer inhibitors persistence who underwent ITI and complete follow-up. Plasma-derived FVIII concentrate was used at 70$180 \mathrm{IU} / \mathrm{kg} /$ day doses. The success of the treatment is defined by achieving a negative titer and a halflife recovery of the FVIII. The results were expressed in median (range). Results: In 13 patients, the inhibitor was identified at an average age of 17.6 months, after 35.2 days of exposure to the FVIII. 11 patients $(84.6 \%)$ recovered the half-life of FVIII after 49.6 months of treatment. In the patients who responded to treatment, the inhibitor titer was negative at 6 months on average. Conclusions: ITI is the treatment of choice for patients with hemophilia A and inhibitors persistence. ITI must be personalized since the time response is variable in each patient.
\end{abstract}

Keywords:

Hemophilia A; immune tolerance induction; inhibitor; factor VIII; neutralizing antibodies

\section{Introducción}

Hemofilia A (HA) es el defecto heredado grave de la coagulación más común y se debe a la deficiencia de Factor VIII (FVIII). La terapia de reemplazo es el tratamiento para prevenir los sangrados recurrentes y para la resolución exitosa de los eventos hemorrágicos en estos pacientes. En la actualidad la aparición de aloanticuerpos neutralizantes (inhibidores) dirigidos contra el FVIII, es la complicación más grave del tratamiento. $\mathrm{Su}$ desarrollo es más frecuente en HA que en hemofilia $\mathrm{B}$, y en pacientes severos con $<1 \%$ FVIII/FIX, en comparación con los pacientes moderados a leves. Esta complicación puede aparecer a cualquier edad, pero se concentra especialmente en niños, y después de las primeras 10 a 15 exposiciones a FVIII exógeno. Se describe una frecuencia general de presentación de un 20$30 \%$ en hemofilia $\mathrm{A}^{1-5}$.

El manejo de los pacientes con hemofilia e inhibidores se enfoca de dos maneras: erradicación de estos anticuerpos, con un tratamiento a largo plazo denominado Inducción de Tolerancia Inmune (ITI). El segundo se refiere al manejo de los eventos agudos hemorrágicos, mientras no se alcance la inmunotolerancia, y que se realiza con los llamados agentes by pass $^{1,5-7}$.

La ITI es, en este momento, la mejor alternativa terapéutica para los pacientes con inhibidor a FVIII exógeno. Garantiza a futuro una terapia de reemplazo eficaz para el tratamiento de eventos hemorrágicos, y permite instaurar regímenes de profilaxis que eviten la aparición de secuelas como la artropatía, mejorando la calidad de vida, y sangrados de riesgo vital. Puede realizarse en cualquier etapa de la vida y consiste en la administración de altas dosis de FVIII, en forma regular por un periodo de meses a años, con el fin de hacer tolerante al sistema inmune frente al antígeno y prevenir en un futuro producción de nuevos anticuerpos. Se describe un éxito de este tratamiento entre $60-80 \%^{8-12}$. El primer reporte de ITI fue en el año 1970 en Alemania en un paciente con Hemofilia A e inhibidores de alto título que, a los 24 meses de ITI, pudo iniciar profilaxis de FVIII, resultando en una mejoría de su estatus osteomuscular y disminución de su morbilidad ${ }^{13}$. A la fecha se han señalado en diferentes publicaciones los factores pronósticos involucrados en el resultado de un tratamiento con inmunotolerancia (tabla 1$)^{14-19}$.

En el Hospital Roberto del Río, centro de referencia nacional de hemofilia en la red pública de Chile, se inició tratamiento de ITI en la población pediátrica con hemofilia A e inhibidores desde el año 2008. Nuestro objetivo fue caracterizar una cohorte de niños portadores de Hemofilia A severa y aloanticuerpos neutralizantes persistentes de alto título tratados con inmunotolerancia. Además, reportamos factores de riesgo relacionados y resultados de este intensivo tratamiento.

Tabla 1. Factores de mal pronóstico sugeridos para resultado de la Inducción de Tolerancia Inmune

Títulos de inhibidor al inicio de ITI > 10 UB

Pico histórico de título > 200 UB

Pico de título durante ITI > 200 UB

Edad al inicio de ITI > 8 años

Latencia de inicio de ITI > 5 años

Interrupción de Inmunotolerancia $>2$ semanas

ITI: Inducción de Tolerancia Inmune, UB: Unidades Bethesda. 


\section{Pacientes y Método}

\section{Diseño del estudio y selección de pacientes}

Estudio retrospectivo, descriptivo de un grupo de pacientes con hemofilia A severa (FVIII $<1 \%$ ) e inhibidores, que ingresaron a régimen de ITI desde septiembre 2008 a septiembre 2018. El protocolo fue aprobado por el Comité de Ética de la Investigación del Servicio Metropolitano Norte. Todos los pacientes incluidos en régimen de tratamiento son altos respondedores, es decir, con títulos de inhibidor en algún momento de la evolución previa a tratamiento $\geq 5 \mathrm{UB} / \mathrm{ml}$. Antes del inicio de la terapia, los padres/tutores fueron informados sobre la envergadura del tratamiento y se les hizo firmar un consentimiento.

\section{Régimen de ITI}

La administración de ITI fue diaria en todos los pacientes en un inicio. Las dosis estuvieron sujetas a disponibilidad de FVIII en el país, con una media de $100 \mathrm{UI} / \mathrm{kg}$. Los concentrados de FVIII utilizados en este tratamiento fueron de origen plasmático, con alto contenido de von Willebrand (pdFVIII/VW), utilizándose el mismo producto que había generado la aparición de inhibidores (Fandhi ${ }^{\circledR}$, Alphanate ${ }^{\circledR}$, ambos de Grifols $\left.{ }^{\circledR}\right)$. Se instaló catéter venoso central para administrar el concentrado dependiendo de la dificultad para garantizar el acceso vascular. La inmunotolerancia comenzó cuando los títulos se encontraban por debajo de $10 \mathrm{UB} / \mathrm{ml}$, como se recomendaba internacionalmente.

\section{Seguimiento de Laboratorio y definición de éxito}

Se realizó control periódico de titulación de inhibidores cada 8 semanas hasta que el valor fue $<0,5 \mathrm{UB} /$ $\mathrm{ml}$. Una vez alcanzado esta negativización del inhibidor se realizó estudio de recuperación de FVIII cada 4 semanas, esperando que a la hora de infusión se obtuviera un nivel de más del 66\% del FVIII en plasma.

Tabla 2. Definiciones de resultado de tratamiento con Inducción de Tolerancia Inmune

Éxito Titulación de inhibidores negativa. Recuperación de vida media de FVIII a las 6 h después de un periodo de lavado de $72 \mathrm{~h}$ Niveles plasmáticos de FVIII > 1\% con dosis de $50 \mathrm{UI} / \mathrm{Kg}$ medidas a las $48 \mathrm{~h}$

Éxito Titulación de inhibidores negativa, sin recuperación de la vida parcial media, sin respuesta anamnestica, manteniendo con FVIII de $>1 \%$ recibiendo dosis diarias. Permite dejar profilaxis

Falla Persistencia de títulos de inhibidores sin mostrar descenso de al menos un 20\% del título durante los 6 meses siguientes posteriores a tres meses de iniciada ITI o falla en alcanzar tolerancia en un plazo de 33 meses

FVIII: Factor VIII, UI: unidades internacionales.
Una vez logrado esto, se dio inicio al estudio de farmacocinética, para establecer recuperación de vida media, cada 8 semanas. Las definiciones de respuesta a ITI se detallaron en la tabla $2^{8,11,18,20}$. En aquellos pacientes que recibían dosis diarias de ITI, una vez que alcanzaron una medición de FVIII a la hora de más de 66\% post infusión de $50 \mathrm{UI} / \mathrm{Kg}$, y tras una recuperación de vida media mayor a $25 \%$, se disminuyó la frecuencia de administración de ITI a días alternos, en consenso con los padres, manteniendo igual dosis, de manera hacer más tolerable este intenso tratamiento.

\section{Tratamiento de los eventos hemorrágicos durante ITI}

Durante el tiempo en que el paciente presentaba cuantificación de inhibidores $\geq$ a $5 \mathrm{UB} / \mathrm{ml}$, se utilizaron agentes by pass: Factor VII recombinante activado (rFVIIa) $120-180 \mathrm{ugr} / \mathrm{Kg} /$ dosis y Complejo protrombínico activado (aPCC) 50-100 UI/Kg/dosis. La frecuencia del tratamiento se evaluó en forma personalizada según respuesta clínica. Si no hubo respuesta clínica con uno de ellos se utilizó el otro agente, y en ocasionalmente se administraron ambos secuencialmente según recomendaciones internacionales ${ }^{21,22}$. Una vez que los pacientes disminuyeron sus títulos de inhibidor a $<$ de 5 UB, se utilizó para los eventos hemorrágicos FVIII en dosis que permitan neutralizar el título de inhibidor, y con frecuencia de cada 4-6 h.

Resultados expresados como media (rango).

\section{Resultados}

Las características demográficas y clínicas antes del inicio del tratamiento se muestran en la tabla 3. Un total de 14 pacientes con hemofilia A e inhibidores persistentes de alto título ingresaron a régimen de ITI. Un paciente abandonó tratamiento a los 9 meses de iniciada, con título de inhibidores aún sobre 5 UB y no siguió contralándose en nuestro centro, siendo el único excluido de análisis. La edad al momento de identificar presencia del inhibidor fue de 17,6 meses (2-48), tras 35,2 días (9-112) de exposición a pdFVIII/VW. En dos pacientes no se obtuvo el dato de días de exposición de factor antes de la aparición de los inhibidores. La edad de inicio de ITI fue de 3,1 años $(0,6-8,0)$ y el tiempo de latencia de la ITI tras identificación de inhibidores fue de 19,6 meses (5,544). Seis pacientes necesitaron instalación de catéter venoso central (Port-a-Cath), de los cuales ninguno completó su tratamiento utilizando esta vía. En 3 pacientes hubo que retirarlo por infección. Dos pacientes utilizaron el primer año catéter percutáneo y el resto vía periférica (por punción directa) para administrar tratamiento desde el inicio. 
Tabla 3. Características clínicas y demográficas de los pacientes tratados con Inducción de Tolerancia Inmune

\begin{tabular}{|c|c|c|c|c|c|c|c|}
\hline Caso & $\begin{array}{c}\text { Edad al Dg } \\
\text { de inhibidor } \\
\text { (meses) }\end{array}$ & $\begin{array}{c}\text { Mutación gen } \\
\text { FVIII }\end{array}$ & $\begin{array}{l}\text { DEA a FVIII } \\
\text { (días) }\end{array}$ & $\begin{array}{l}\text { Título al Dg } \\
\text { (UB) }\end{array}$ & $\begin{array}{c}\text { Edad al inicio } \\
\text { de ITI } \\
\text { (años) }\end{array}$ & $\begin{array}{c}\text { Latencia de } \\
\text { inicio de ITI } \\
\text { (meses) }\end{array}$ & $\begin{array}{c}\text { Pico título } \\
\text { histórico } \\
\text { pre-ITI }\end{array}$ \\
\hline 1 & 13 & Frameshift & 16 & 0,6 & 2,01 & 12,13 & 7,2 \\
\hline 2 & 32 & Inv int 22 & 79 & 10 & 4,31 & 21 & 10 \\
\hline 3 & 19 & Inv int 22 & 16 & 18 & 1,94 & 6 & 18 \\
\hline 4 & 19 & Nonsense & 112 & 40 & 4,19 & 32,4 & 40 \\
\hline 5 & 2 & Inv int 22 & 20 & 16,2 & 0,59 & 5,5 & 16,2 \\
\hline 6 & 16 & Inv int 22 & $\mathrm{D}$ & 26 & 3,51 & 14,4 & 26 \\
\hline 7 & 4 & Del exón & 9 & 4,8 & 1,08 & 10,2 & 17,6 \\
\hline 8 & 17 & Inv int 22 & 20 & 146 & 3,51 & 10,2 & 146 \\
\hline 9 & 15 & Nonsense & 50 & 140 & 2,7 & 25,6 & 146 \\
\hline 10 & 17 & Del exón & 12 & 42 & 2,8 & 17,1 & 143 \\
\hline 11 & 15 & Inv int 22 & 30 & 8,4 & 4,1 & 34,6 & 8,4 \\
\hline 12 & 12 & Inv int 22 & 25 & 384 & 1,59 & 8,3 & 384 \\
\hline 13 & 18 & Inv int 22 & 42 & 1.240 & 4,2 & 33,6 & 1.240 \\
\hline 14 & 48 & Inv int 22 & D & 13,5 & 8 & 44 & 54 \\
\hline
\end{tabular}

Dg: diagnóstico, DEA: días acumulados de exposición, D: desconocida, FVIII: factor VIII, ITI: Inducción de Tolerancia Inmune, UB: unidades Bethesda, Inv int 22: Inversión intron 22, Del exón: Deleción exón.

La descripción de los aspectos más importantes de tratamiento de ITI por pacientes se detallan en la tabla 4. Todos los pacientes iniciaron su tratamiento con valores menores a $10 \mathrm{UB}$, y las dosis utilizadas fueron en un rango de 70-180 UI/Kg/día. Siete pacientes pudieron pasar a esquema de ITI de días alternos. Dos pacientes suspendieron al año la terapia por decisión de los padres. Ambos se encontraban con una titulación de inhibidores negativa al momento del abandono, pero sin haber alcanzado normalización de vida media del FVIII. Ambos reinician ITI 18 meses más tarde por episodios hemorrágicos frecuentes, y aunque el título del inhibidor había ascendido se mantuvo en $<$ de 5 UB. Se utilizó para este nuevo curso de tratamiento el mismo concentrado de $\mathrm{pdFVIII/VW,} \mathrm{con} \mathrm{dosis}$ de aproximadamente $86-88 \mathrm{UI} / \mathrm{Kg}$, distanciándose la frecuencia a tres veces a la semana. Ambos alcanzan la normalización de la vida media a los 20 y 24 meses después. La profilaxis con agentes by pass durante la ITI la recibieron sólo 2 pacientes con fenotipo hemorrágico. Respecto a la distribución de anomalía genética del FVIII, las mutaciones encontradas corresponden todas a mutaciones nulas, las cuales se describen con mayor frecuencia de aparición de inhibidores.

De los 13 pacientes con evaluación completa, 2 no respondieron a la ITI y 11 recuperaron la vida media del FVIII en 49,6 meses (26-70), por lo que el éxito de ITI en esta serie fue de $84,6 \%$. En los pacientes que res- pondieron, el título del inhibidor se negativizó en una media de 7,3 (1-20) meses, lo que permitió suspender tempranamente el uso de agentes by pass.

De los 11 niños que lograron inmunotolerancia, ninguno presentó un título histórico > 200 UB a diferencia de los 2 pacientes que fracasaron, los cuales en algún momento de su vida presentaron valores mayores al corte de riesgo. Todos los pacientes iniciaron su tratamiento con $<10$ UB y todos con menos de 8 años de edad. El tiempo transcurrido entre el diagnóstico de inhibidores e inicio de ITI mayor a 24 meses ocurrió en tres pacientes, uno de los cuales fue fracaso.

En todos los episodios hemorrágicos durante el periodo de tratamiento de ITI se utilizó rFVIIa y/o aPCC, según antecedentes de respuesta con uno u otro de estos agentes en episodios de sangrados anteriores. En todos los eventos se alcanzó respuesta y no hubo complicaciones trombóticas. Sólo hubo un evento grave, una hemorragia intracraneana en uno de los pacientes a los 2 meses de iniciada la inmunotolerancia. Se manejó con rFVIIa con buena respuesta quedando sin secuelas.

Una vez finalizada la ITI exitosamente, todos los menores pasaron a régimen de profilaxis con pdFVIII/ VW a días alternos o tres veces a la semana dependiendo de su farmacocinética. El descenso de la dosis fue paulatino, hasta alcanzar el régimen de profilaxis habitual para los pacientes con hemofilia A severa de 
Tabla 4. Seguimiento y resultados de los pacientes tratados con Inducción de Tolerancia Inmune

\begin{tabular}{|c|c|c|c|c|c|c|}
\hline Caso & $\begin{array}{c}\text { Título mayor } \\
\text { durante ITI } \\
\text { (UB) }\end{array}$ & $\begin{array}{l}\text { Título inicio } \\
\text { ITI } \\
\text { (UB) }\end{array}$ & $\begin{array}{c}\text { Dosis ITI } \\
\text { (Ul/Kg/dosis) }\end{array}$ & $\begin{array}{c}\text { Tiempo para negativizar UB } \\
\text { tras iniciar ITI } \\
\text { (meses) }\end{array}$ & $\begin{array}{c}\text { Tiempo recuperación de vida } \\
\text { media desde inicio de ITI } \\
\text { (meses) }\end{array}$ & $\begin{array}{l}\text { Resultado de } \\
\text { tratamiento }\end{array}$ \\
\hline 1 & 6,2 & 7,2 & 130 & 1 & $26^{+}$ & $\mathrm{E}$ \\
\hline 2 & 30 & 3 & 88 & 4 & $60^{*}$ & $E$ \\
\hline 3 & 110 & 1,3 & 154 & 8 & 42 & $\mathrm{E}$ \\
\hline 4 & 18,3 & 5 & 86 & 15 & $52^{+}$ & $E$ \\
\hline 5 & 11,9 & 1,7 & 136 & 2 & $55^{+}$ & $E$ \\
\hline 6 & 36,9 & 3,5 & 70 & 12 & $70^{+}$ & $E$ \\
\hline 7 & 76 & 1,2 & 180 & 3 & $26^{+}$ & E \\
\hline 8 & 140 & 3,5 & 86 & 20 & $59^{*}$ & $E$ \\
\hline 9 & 82 & 2 & 76 & 6 & 39 & $E$ \\
\hline 10 & 176,4 & 0 & 75 & 4 & $66^{+}$ & $\mathrm{E}$ \\
\hline 11 & 19 & 1,1 & 107 & 5 & $51^{+}$ & $E$ \\
\hline 12 & 1.190 & 9 & 108 & $S / R$ & $S / R$ & $F$ \\
\hline 13 & 50 & 2,1 & 100 & $S / R$ & $S / R$ & $\mathrm{~F}$ \\
\hline 14 & 300 & 4 & 100 & $\mathrm{NE}$ & $\mathrm{NE}$ & $\mathrm{NE}$ \\
\hline
\end{tabular}

ITI: Inducción de Tolerancia Inmune, UB: Unidad Bethesda, NE: No evaluado por abandono precoz de ITI, S/R: sin respuesta, E: éxito, F: fracaso. +Modificación frecuencia de ITI una vez alcanzada parcialmente vida media. *Abandono de tratamiento y reinicio de ITI.

20-30 UI/Kg dosis, asegurando un nivel de FVIII $>$ de $1,5 \%$ a las 48 o $72 \mathrm{~h}$.

No se ha presentado recaída en los pacientes que han finalizado su tratamiento de ITI.

\section{Discusión}

La erradicación de los inhibidores es el tratamiento estándar en todo el mundo para los pacientes con Hemofilia A e inhibidores. Los distintos esquemas de ITI utilizados tienen como finalidad generar el reconocimiento como propio del FVIII infundido, permitiendo tratamientos efectivos con el concentrado de factor deficitario, además de poder implementar regímenes de profilaxis. La decisión del esquema de tratamiento depende de distintas variables. Primero es el acceso a tratamiento, es decir la cantidad disponible de concentrado de FVIII que tiene el centro de atención o el país; el segundo la disposición de los padres a seguir un tratamiento tan intensivo; y en tercer lugar, está la disponibilidad de adecuado acceso vascular. En pacientes con malos accesos venosos, como lactantes y niños pequeños, puede ser necesario el uso de catéter venoso central de larga duración, con todas las aristas que esta elección conlleva. La diferencia entre estos distintos regímenes se traduce en el tiempo que nos demoramos en alcanzar una vida media normal del FVIII. Con un régimen de altas dosis alcanzaremos una inmunotolerancia en un periodo de tiempo más corto, razón por la cual actualmente es la recomendación en aquellos pacientes con fenotipo muy hemorrágico. Esta última recomendación sería una cuarta variable a considerar para la elección de esquema de ITI en aquellos países en los cuales se cuenta con los recursos para llevarlo a cabo. Nosotros utilizamos un esquema de dosis variables ajustadas al peso del niño, a la presentación del producto (los viales de mayor concentración con que se cuenta son de $1.000 \mathrm{UI} / 10 \mathrm{ml}$ ), al acceso venoso, y a la disponibilidad del concentrado en el país.

Dentro de los factores que se plantean como de buen pronóstico de ITI, uno de los más potentes ha sido el inicio de ITI con menos de 10 UB y un pico histórico de inhibidor $<200$ UI. Respecto a nuestra cohorte, podemos decir que los dos pacientes que no respondieron a ITI después de dos años de tratamiento intensivo fueron aquellos que presentaron un título histórico > $300 \mathrm{UB}$, ya que el resto de las variables involucradas están distribuidas sin diferencia en el resto de los pacientes, y todos iniciaron su tratamiento con un título de menos de 10 UB.

Pudimos notar en nuestra cohorte, que la modificación del esquema de inmunotolerancia una vez iniciada, no repercute en el resultado final. Tras alcanzar títulos negativos y una recuperación de vida media de $25 \%$, pudimos modificar el régimen de tratamiento $y$, 
además, manejar con dosis altas de FVIII los episodios hemorrágicos y cirugías intercurrentes. Tener cierta flexibilidad permite tolerar mejor el tratamiento por parte del paciente y de la familia.

El éxito de este tratamiento alcanzó un 84,6\%, lo que nos parece un muy buen resultado al compararlo con publicaciones internacionales previas, y creemos que se debe, en parte, al uso de concentrados de origen plasmático. Fundamentamos nuestra observación a la luz de los resultados descritos por el estudio SIPPET, único estudio prospectivo, randomizado que compara la inmunogenicidad de los concentrados de FVIII plasmático versus FVIII recombinante ${ }^{23}$. Este evidenció que el uso de concentrados de origen plasmático, no solo mostró una tasa de inhibidores de alto título menor, sino también que, cuando estos se presentaron, el pico histórico fue más bajo. Ambas variables determinan, como mencionamos en la tabla 1 , factores de mejor pronóstico. Por otro lado, el beneficio del uso de pdFVIII/VW, esta descrito como tratamiento de rescate en aquellas ITI que han fallado con el uso de FVIII recombinante, lo que reforzaría la observación antes expuesta ${ }^{24}$.

Para el manejo de las hemorragias agudas, mientras no exista respuesta al factor deficitario está la terapia by pass: rFVIIa y aPCC. El mecanismo de acción de estos dos concentrados radica en potenciar los procesos de hemostasia por vías alternativas. Sin embargo, presentan varios inconvenientes como son una respuesta impredecible, lo que puede llevar a hemorragias intratables con riesgo vital, o determinar importantes secuelas. Además, no hay un examen de laboratorio con el cual se pueda realizar un seguimiento, por lo tanto, la efectividad de tratamiento sólo se mide clínicamente. Por último, los costos de esta terapia superan en forma significativa la terapia clásica de reemplazo ${ }^{25}$. Recientemente se ha aprobado el uso de un anticuerpo monoclonal para esquema de profilaxis en hemofilia A e inhibidores con muy buena respuesta, que se traduce en una disminución significativa del número de eventos hemorrágicos. La aparición de esta nueva herramienta terapéutica está siendo debatida, y sería una alternativa propuesta como profilaxis en aquellos pacientes con inhibidores que tienen fenotipo hemorrágico, mientras alcanzan la inmunotolerancia. En nuestra opinión, consideramos que este nuevo tratamiento no reemplaza la ITI, dado que no es útil para el manejo de eventos hemorrágicos agudos, en los cuales se deberá utilizar agentes by pass. Sabemos además que el mejor escenario para un paciente con inhibidores es su erradicación, y poder utilizar el factor deficitario en dosis y frecuencias habituales.

Pese a las limitaciones propias de una cohorte de casos pequeña, además de las limitaciones propias de un estudio descriptivo, creeemos que nuestros resultados son valiosos, ya que corresponden al seguimiento de 10 años de un tratamiento complejo, que permite eliminar la mayor complicación actual en el tratamiento de los pacientes con hemofilia, con una elevada tasa de respuesta.

\section{Conclusión}

La ITI es el tratamiento de elección para los pacientes que padecen hemofilia A e inhibidores. Los resultados de nuestra cohorte de pacientes tratados con pdFVIII/VW son muy buenos, permitiendo la negativización del inhibidor en un período cercano a 7,3 meses de iniciado el tratamiento y un éxito final cercano a $85 \%$. La inmunotolerancia debe personalizarse y el tiempo de respuesta es variable en cada paciente. Un título de inhibidores mayor a 200 UB puede ser considerado como un umbral de mala respuesta.

Agradecimientos: Dra Viviana Abarca Villaseca y Dra Claudia Carmona Silva por el apoyo en el tratamiento de algunos de los pacientes de esta serie.

\section{Responsabilidades Éticas}

Protección de personas y animales: Los autores declaran que los procedimientos seguidos se conformaron a las normas éticas del comité de experimentación humana responsable y de acuerdo con la Asociación Médica Mundial y la Declaración de Helsinki.

Confidencialidad de los datos: Los autores declaran que han seguido los protocolos de su centro de trabajo sobre la publicación de datos de pacientes.

Derecho a la privacidad y consentimiento informado: Los autores han obtenido el consentimiento informado de los pacientes y/o sujetos referidos en el artículo. Este documento obra en poder del autor de correspondencia.

\section{Conflicto de intereses}

Los autores declaran no tener conflicto de intereses. 


\section{Referencias}

1. Di Michele DM. Inhibitors: resolving diagnostic and therapeutic dilemmas. Haemophila 2002;8:280-7.

2. Kreuz W, Ettingshausen CE, Auerswald $\mathrm{G}$ et al. Epidemiology of inhibitors and current treatment strategies. Haematologica 2003; 88: EREP04.

3. Wight J, Paisley S. The epidemiology of inhibitors in haemophilia A: a systematic review. Haemophilia. 2003;9(4):418-35.

4. Gouw SC, van den BergHM, Fischer K, Auerswald G, Carcao M, Chalmers E, et al. Intensity of factor VIII treatment and inhibitor development in children with severe hemophilia A: the RODIN study. Blood. 2013; 121(20):4046-55.

5. Carcao M, Goudemand J. Inhibitors in Haemophilia: A primer. Fifth edition educational materials www.wfh.org

6. Abshire T, Kenet G. Recombinant factor VIIa: review of efficacy, dosing regimens and safety in patients with congenital and acquired factor VIII or IX inhibitors. J Thromb Haemost 2004; 2: 899-909.

7. Astermark J, Donfield SM, DiMichele $\mathrm{DM}$ et al. A randomized comparison of bypassing agents in hemophilia complicated by an inhibitor: the FEIBA NovoSeven Comparative (FENOC) Study. Blood 2007; 109:546-51.

8. Di Michele DM, Hoots K, Pipe SW, Rivard GE, Santagostino E. International workshop on immune tolerance induction: consensus recommendations. Haemophilia 2007;13(Suppl. 1), 1-22.

9. Wight J, Paisley S, Knight C. Immune tolerance induction in patients with haemophilia A with inhibitors: a systematic review. Haemophilia 2003; 9(4): 436-63.

10. Valentino LA, Kempton CL, KruseJarres R, Mathew P, Meeks SL. Reiss UM; International Immune Tolerance
Induction Study Investigators.US Guidelines for immune tolerance induction in patients with haemophilia A and inhibitors. Haemophilia. 2015; 21:559-67.

11. Collins PW, Chalmers E, Hart DP, et al. Diagnosis and treatment of factor VIII and IX inhibitors in congenital haemophilia: (4th edition) A United Kingdom Haemophilia Centre Doctors' Organisation guideline approved by the British Committee for Standards in Haematology. Brit J Haematol. 2013; 162:758-73.

12. Gringeri A, Mannucci PM. Italian guidelines for the diagnosis and treatment of patients with haemophilia and inhibitors. Haemophilia. 2005; 11:611-9.

13. Brackmann HH, Gormsen J. Massive factor-VIII infusion in haemophiliac with factor-VIII inhibitor, high-responder. Lancet. 2(8044), 933 (1977).

14. Mariani G, Ghirardini A, Bellocco R. Immune tolerance in hemophiliaprincipal results from the International Registry Report of the factor VIII and IX Subcommittee. Thromb Haemost 1994; 72: 155-8.

15. DiMichele DM, Kroner BL. The North American Immune Tolerance Registry: practices, outcomes, outcome predictors. Thromb Haemost 2002; 87: 52-7.

16. Lenk H. The German Registry of immune tolerance treatment in hemophilia-1999 update. Haematologica 2000;85:45-7.

17. Haya S, Lopez MF, Aznar JA, Batlle J. Immune tolerance treatment in haemophilia patients with inhibitors: the Spanish Registry. Haemophilia 2001;7:154-9.

18. Hay CR, DiMichele DM. The principal results of the International Immune Tolerance Study: a randomized dose comparison. Blood 2012;119:1335-44.
19. Coppola A, Margaglione M, Santagostino $\mathrm{E}$ et al. Factor VIII gene (F8) mutations as predictors of outcome in immune tolerance induction of hemophilia A patients with high-responding inhibitors. J Thromb Haemost 2009;7:1809-15.

20. Collins, P, Chalmers E, Alamelu J, Hay $\mathrm{C}$ et al. First-line immune tolerance induction for children with severe haemophilia A: A protocol from the UK Haemophilia Centre Doctors' Organisation Inhibitor and Paediatric Working Parties. Haemophilia. 2017; 23:654-59.

21. Schneiderman J, Nugent DJ, Young G. Sequential therapy with activated prothrombin complex concentrate and recombinant factor VIIa in patients with severe haemophilia and inhibitors. Haemophilia 2004;10:347-51.

22. JTeitel J, Berntorp E, Collins P, et al. A systematic approach to controlling problem bleeds in patients with severe congenital haemophilia A and high-titre inhibitors. Haemophilia 2007;13: 256-63.

23. Peyvandi F, Mannucci PM, Garagiola I, El-Beshlawy A, Elalfy M, Ramanan V et al. A randomized trial of factor VIII and neutralizing antibodies in hemophilia A. New England Journal of Medicine, 2016; 374(21):2054-64.

24. Oldenburg J, Jiménez-Yuste V, PeiróJordán R, Aledort LM, Santagostino E. Primary and rescue immune tolerance induction in children and adults: a multicentre international study with a VWF-containing plasma-derived FVIII concentrate. Haemophilia. 2014; 20 Suppl 6:17-26.

25. D'Angiolella LS, Cortesi PA, Rocino A, Coppola A, et al. The socioeconomic burden of patients affected by hemophilia with inhibitors. Eur J Haematol. 2018;101(4):435-56. 